\title{
An inviscid model for the vortex-street wake
}

\author{
By P. G. SAFFMAN AND J. C. SCHATZMAN \\ Applied Mathematics, California Institute of Technology, Pasadena, California 91125
}

(Received 13 August 1981 and in revised form 21 December 1981)

An inviscid model for the Kármán vortex street, containing vortices of uniform vorticity surrounced by irrotational fluid, is related to the wake behind a bluff body by a global analysis requiring the conservation of momentum, energy and vorticity. Some comparison is made with experimental results reported in the literature. A qualitative procedure is proposed whereby the slow evolution of the wake through viscous effects is approximated. Some comments are made regarding the relevance of the stability properties of the inviscid street. Some calculations are made for the 'secondary vortex street' that is observed after breakdown and rearrangement, and comparison is made with experiment.

\section{Introduction}

In previous papers (Saffman \& Schatzman 1981, 1982; Schatzman 1981), a model for the Kármán vortex street was described which is similar to von Kármán's point vortex model (von Kármán 1911, 1912; von Kármán \& Rubach 1912), except that the vortices were assumed to be of finite area and uniform vorticity. Numerical solutions of the Euler equations for this model were obtained and properties of the street were calculated.

In this paper, the equations are derived that permit relating the properties of the street (vortex strength, size, and spacing) to three parameters describing the local behaviour of the flow in the vicinity of a blunt body producing the street. The first parameter is the drag, the second specifies the rate of shedding of vorticity from the body that ends up in the wake, and the third describes the energy loss through viscous dissipation in the near wake. It is observed that values of these parameters more or less in agreement with experiment lead to predictions of the model which are also compatible with observations. Further, by permitting these parameters to vary slowly with time, i.e. by allowing a slow loss of vorticity and energy of the wake, the evolution of the vortex street through viscous effects is simulated. Some comments are also made regarding the relevance of the stability properties of the inviscid street, rough calculations are made describing the 'secondary vortex street' that is observed after breakdown and rearrangement, and comparison is made with experiment.

Similar semi-empirical calculations have been done by Roshko (1954) and Weihs (1973). However, both researchers used the point-vortex approximation and assumed von Kármán's value for the spacing ratio, $\kappa=0 \cdot 28055$. While the former approximation is probably reasonable (as will be discussed later), the latter assumption is probably not, and it is certainly not compatible with a slow evolution of the street. Weihs claims to have modelled the vortex pairing observed by Taneda (1959) and others, but the analysis assumes constant wake frequency (Strouhal number) and is hence apparently not relevant to this phenomenon.

It is recognized that a purely inviscid model such as the one proposed is not completely adequate for making accurate predictions for the real-fluid phenomena. 
Nevertheless, the purpose of this paper is to study, in some detail, the characteristics of the approximating inviscid flow in order to see to what extent the properties of the real flow can be reproduced and predicted.

In $\$ 2$, we derive expressions for the momentum, energy, and vorticity flux in an inviscid uniform vortex street of finite-area vortices placed in a uniform stream of velocity $U_{0}$. These are expressed in terms of the strength and size of the vortices, the distance between them, and $U_{0}$. These fluxes are related to the drag on the body, the amount of kinetic energy that is lost in the near wake by dissipation, and the amount of vorticity that is shed by the body. In this way, closed equations are obtained for the properties of the street in terms of quantities determined by the flow field close to the body. These equations are solved numerically in $§ 3$ and the predicted street properties are shown to correspond reasonably well with observations.

In $\$ 4$, the slow evolution of the street is considered by allowing for slow dissipation of energy and annihilation of vorticity. In this way, predictions are made for variations of the spacing ratio, again in reasonable agreement with experiment.

The question of the street stability is considered in $§ 5$. It is shown that the initially small growth rates of infinitesimal disturbances become large as the street evolves, and this can account for the observed instability of the street after some number of periods. Finally, in $\$ 6$, we consider to what extent the inviscid model can predict or is consistent with the sometimes-observed rearrangement.

\section{Conservation equations}

The assumptions of the model are that the flow is incompressible, inviscid, two-dimensional, spatially periodic in the streamwise direction, and irrotational outside vortices of uniform vorticity which lie in the well-known Kármán vortex-street pattern. The inherent assumption that the street does not evolve with time, and therefore that the fully infinite vortex-street model is applicable, permits an analytical analysis of momentum and energy conservation in the flow past a body. The method is to consider the conservation equations for the fluid contained in a large box surrounding the body and cutting the wake far downstream (see figure 1), to let the box expand to infinity, and to time-average the equations over one period of the wake oscillation. The details of the analysis follow.

Momentum conservation may be studied in a manner similar to that employed for the point vortex street (see e.g. Goldstein 1938). A slightly different argument proceeds as follows: Consider a co-ordinate system fixed with respect to the street, as in figure 1 , in which the unsteady motion is concentrated in the vicinity of the body (which travels upstream with speed $U_{0}-U_{\mathrm{s}}$ ) and the speed at infinity is $U_{\mathrm{s}}$. The effect of the body on the flow field can be modelled by an equivalent distribution of forces, concentrated in the body, so that the equation of motion can be written (with unit fluid density)

$$
\frac{\partial \mathbf{u}}{\partial t}+\mathbf{u} \cdot \nabla \mathbf{u}=-\nabla p-\mathbf{F} .
$$

This equation is valid throughout space (including the region of fictitious fluid in which the body lies), where $F=0$ outside of the body region, and where the integral of $F$ over the body region gives the force on the body. The vorticity $\omega$ then satisfies

$$
\frac{\partial \omega}{\partial t}+\mathbf{u} \cdot \nabla \omega=-\nabla \times \mathbf{F}
$$




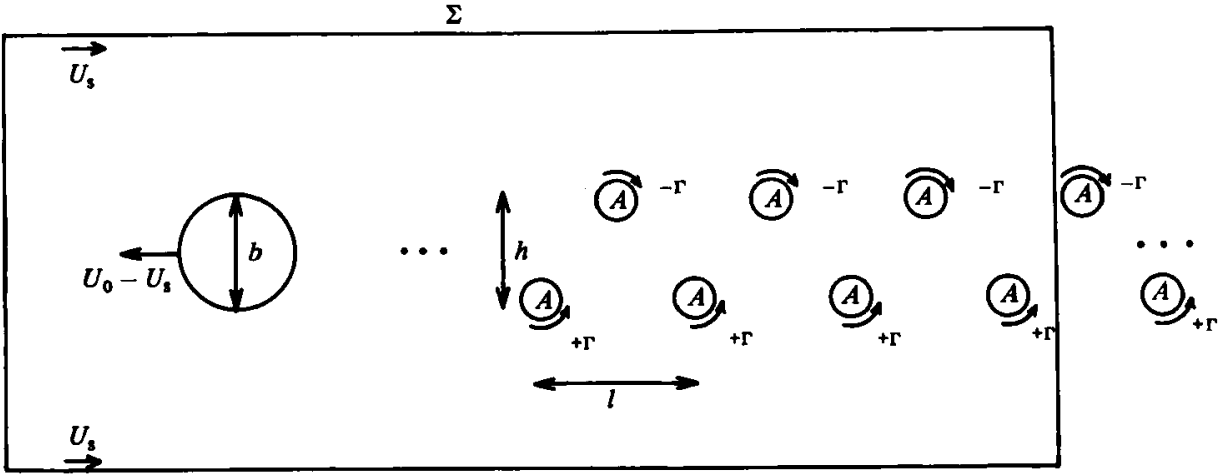

Figure 1. Sketch of the flow configuration in a co-ordinate system fixed relative to the street.

Flow is most unsteady near the body.

A fixed region $\Sigma$ is considered that encloses the body and part of the street. Then

$$
\frac{\partial}{\partial t} \int_{\Sigma}(\mathbf{x} \times \omega) d \sigma=\int_{\Sigma}\left(\mathbf{x} \times \frac{\partial \omega}{\partial t}\right) d \sigma=-\int_{\Sigma} \mathbf{x} \times(\nabla \times \mathbf{F}) d \sigma-\int_{\Sigma} \mathbf{x} \times(\mathbf{u} \cdot \nabla) \omega d \sigma
$$

on using (2.2). This can easily be shown to equal the time rate of change of momentum within $\Sigma$ in the limit as $\Sigma$ extends to infinity in all directions. Some identities are employed, easily verified, that in two dimensions, for $\mathbf{F}$ of finite extent, incompressible fluid and $\omega$ zero on the boundary of $\Sigma$.

$$
\begin{gathered}
\int_{\Sigma} \mathbf{x} \times(\nabla \times \mathbf{F}) d \sigma=\int_{\Sigma} \mathbf{F} d \sigma=\mathbf{D} \\
\int_{\Sigma} \mathbf{u} \times \omega d \sigma=-\int_{\Sigma} \mathbf{x} \times(\mathbf{u} \cdot \nabla \omega) d \sigma=\oint_{\Sigma}\left[\frac{1}{2} \mathbf{u}^{2} \mathbf{n}-\mathbf{u}(\mathbf{u} \cdot \mathbf{n})\right] d s
\end{gathered}
$$

Further, we write

$$
\mathbf{u}^{\prime}=\mathbf{u}-\mathbf{U}_{\mathrm{s}}, \quad \mathbf{U}_{\mathrm{s}}=U_{\mathrm{s}} \mathbf{x},
$$

so that $\mathbf{u}^{\prime}$ denotes the velocity relative to the free stream. Then (2.3) gives

$$
\frac{\partial}{\partial t} \int_{\Sigma} \mathbf{x} \times \omega d \sigma=-\mathbf{D}+\mathbf{D}^{\prime}-\mathbf{U}_{\mathrm{s}} \times \int_{\Sigma} \omega d \sigma,
$$

where

$$
\mathbf{D}^{\prime}=\oint_{\Sigma}\left[\frac{1}{2} \mathbf{u}^{\prime 2} \mathbf{n}-\mathbf{u}^{\prime}\left(\mathbf{u}^{\prime} \cdot \mathbf{n}\right)\right] d s,
$$

and it is to be remembered that the boundary must not pass through regions containing vorticity. Furthermore, in the limit as the region $\Sigma$ extends to infinity, it is easily seen that the integral may be taken over only the downstream boundary of $\Sigma$ and the velocity field $u^{\prime}=\left(u^{\prime}, v\right)$ may be taken to be that due to a fully infinite vortex street.

The next step is to take the streamwise component of (2.7) and average over one period of the wake oscillation. The last term does not contribute as it is transverse to the stream. The $x$-component of $\mathbf{D}^{\prime}$ can be written, since $u^{\prime}-i v$ is an analytic function of $z \equiv x+i y$,

$$
D^{\prime}=-\frac{1}{2} \operatorname{Im} \int_{-i \infty}^{+i \infty}\left(u^{\prime}-i v\right)^{2} d z,
$$


where the contour is any path going from below to above the street which does not pass through a vortex.

Over one period of the wake oscillation, i.e. over time $l /\left(U_{0}-U_{\mathrm{s}}\right)$, the body travels upstream distance $l$ and two more vortices are created inside $\Sigma$; by assumption, the flow in $\Sigma$ remains otherwise the same. This gives an additional $x$-component contribution to the integral on the left-hand side of $(2.7)$ of amount $-\Gamma h$, where $h$ measures the $y$-separation of the centres of vorticity of the two rows, and $\Gamma$ is the strength of the vortices. Hence, the average over one period gives

$$
\hat{\mathbf{x}} \cdot \overline{\frac{\partial}{\partial t} \int_{\Sigma} \mathbf{x} \times \omega d \sigma}=\frac{U_{0}-U_{\mathrm{s}}}{l}(-\Gamma h) .
$$

Thus, the $x$-component of the drag, averaged over one period (and omitting special symbols for average quantities), is given by

$$
D=\frac{\Gamma h}{l}\left(U_{0}-U_{\mathrm{s}}\right)-\frac{1}{2} \operatorname{Im} \int_{-i \infty}^{+i \infty}\left(u^{\prime}-i v\right)^{2} d z,
$$

where the integral is obtained from the fully infinite vortex street, and the contour passes between neighbouring vortices (it is independent of path, subject to this condition).

This result relating the drag on the body to properties of the infinite street is well known for point vortices, and our object here is to show that it remains correot for vortices of finite size. Note that the argument does not work for the fluctuating lift on the body, which according to (2.7) depends crucially on the fluctuations of circulation and hence on the detailed flow near the body. It was pointed out to us by Professor A. Roshko that a relation between the properties of the street and the fluctuating lift cannot exist because there can be no lift on a flat plate normal to an inviscid flow (assuming the flow separates smoothly at the edges) but there can be a fully developed vortex-street wake.

An extension to (2.11) deals with the case where the street is slowly varying. In (2.6), pick $U_{\mathrm{s}}$ to be the value in the vicinity of the downstream boundary of $\Sigma$; then (2.7) holds exactly. It is assumed that the variation in vortex spacing and size is sufficiently gradual that at each point in the street, far downstream of the body, the propagation velocity $U_{\mathrm{s}}$ and the integral $(2.9)$ for $D^{\prime}$ are given to arbitrary accuracy by the corresponding quantities for the steady uniform street. It may be of use to consider a piecewise uniform street where the downstream boundary of $\Sigma$ is far from the edges of the uniform section in which it lies. To this approximation, (2.8) still leads to (2.9). Furthermore, since the street is assumed to 'age' at a uniform rate, (2.10) also holds, where the quantities on the right-hand side are those for the downstream boundary of $\Sigma$, and hence (2.11) is still approximately correct. The condition that the right-hand side of (2.11) be constant throughout the wake is thus approximately equivalent to the condition that momentum be conserved in the wake.

We now derive a second relation by considering the energy balance between the kinetic energy of the flow in the street and the work done by the body. Multiplying (2.1) by $u$ and integrating gives

$$
\frac{\partial}{\partial t} \int_{\Sigma} \frac{1}{2} \mathbf{u}^{2} d \sigma=D\left(U_{0}-U_{\mathrm{s}}\right)-\oint_{\Sigma}\left(p+\frac{1}{2} \mathbf{u}^{2}\right) \mathbf{u} \cdot \mathbf{n} d s
$$


Substituting $\mathbf{u}=\mathbf{u}^{\prime}+\mathbf{U}_{\mathbf{s}}$, this becomes

$$
\begin{aligned}
\frac{\partial}{\partial t} \int_{\Sigma} \frac{1}{2} \mathbf{u}^{\prime 2} d \sigma- & D U_{0}+\oint_{\Sigma}\left(p+\frac{1}{2} \mathbf{u}^{\prime 2}\right)\left(\mathbf{u}^{\prime} \cdot \mathbf{n}\right) d s+\mathbf{U}_{\mathbf{s}} \cdot \oint_{\Sigma} \frac{1}{2} \mathbf{u}^{\prime 2} \mathbf{n} d s \\
& =-\mathbf{U}_{\mathbf{s}} \cdot\left\{\mathbf{D}+\frac{\partial}{\partial t} \int_{\Sigma} \mathbf{u}^{\prime} d \sigma+\oint_{\Sigma}\left[p \mathbf{n}+\mathbf{u}^{\prime}\left(\mathbf{u}^{\prime} \cdot \mathbf{n}\right)+\mathbf{u}^{\prime}\left(\mathbf{U}_{\mathbf{s}} \cdot \mathbf{n}\right)\right] d s\right\}
\end{aligned}
$$

It follows from integrating (2.1) that the right-hand side of (2.13) is zero.

The Bernouilli equation, valid outside the vortices, gives

$$
p+\frac{1}{2} \mathbf{u}^{\prime 2}+\mathbf{u}^{\prime} \cdot \mathbf{U}_{\mathbf{s}}+\frac{\partial \phi}{\partial t}=0 .
$$

Substituting for $p+\frac{1}{2} \mathbf{u}^{\prime 2}$ in (2.13) and observing that

$$
\oint_{\Sigma} \frac{\partial \phi}{\partial t} \mathbf{u}^{\prime} . \mathbf{n} d s \rightarrow 0
$$

in the limit of large $\Sigma$, since the integrand vanishes inside the wake and each term is at most $O\left(r^{-1}\right)$ outside the wake, where the effect of the body is that of a moving source fed by the wake inflow, the following result is obtained:

$$
D U_{\mathbf{0}}=\frac{\partial}{\partial t} \int_{\Sigma} \frac{1}{2} \mathbf{u}^{\prime 2} d \sigma+\mathbf{U}_{\mathbf{s}} \cdot \oint_{\Sigma}\left[\frac{1}{2} \mathbf{u}^{\prime 2} \mathbf{n}-\mathbf{u}^{\prime}\left(\mathbf{u}^{\prime} . \mathbf{n}\right)\right] d s .
$$

Combining (2.11) and (2.16) then gives

$$
D U_{0}=\frac{\partial}{\partial t} \int_{\Sigma} \frac{1}{2} \mathbf{u}^{\prime 2} d \sigma+U_{\mathrm{s}}\left[D-\frac{\Gamma h}{l}\left(U_{0}-U_{\mathrm{s}}\right)\right] .
$$

In one period, the kinetic energy inside $\Sigma$ is increased by $T l$, where $T$ is the average kinetic energy per unit length over streamwise distance $l$ for the fully infinite street, so that

$$
\overline{\frac{\partial}{\partial t} \int_{\Sigma} \frac{1}{2} \mathbf{u}^{\prime 2} d \sigma}=T l \frac{U_{0}-U_{\mathrm{s}}}{l}
$$

With (2.17) this gives the relation

$$
D=T-\frac{\Gamma h}{l} U_{\mathrm{s}}
$$

For point vortices, $T=\infty$, and the relation is meaningless. This, of course, is one of the objections to using a street of point vortices as a model of the wake. Schlayer (1928) and Kaufmann (1951) used (2.19) without the last term (i.e. they just assumed $D=T$ ) to estimate the size of the vortices, but we are unaware of other attempts to use energy conservation, and the relation (2.19) appears to be new. However, it does depend on the assumption that all the energy of the flow near the body does go into the street and that none is lost by viscous dissipation. It is perhaps more realistic to suppose that a fraction $\epsilon$ of the work done by the body on the fluid is lost. Then $D$ in $(2.12)$ is replaced by $(1-\epsilon) D$, and repeating the argument consistently gives, instead of (2.19),

$$
(1-\epsilon) D=T-\frac{\Gamma h}{l} U_{\mathrm{s}}
$$

Note that viscous dissipation does not affect the momentum-balance equation (2.11) or the vanishing of the right-hand side of (2.13) as momentum is still conserved in 
the absence of boundaries at rest. The effects of dissipation in the far downstream wake would produce a small contribution to $\partial \phi / \partial t$ in (2.15), but this contribution is neglected, although this approximation may not be good. For this reason, the later calculations of vortex area based on (2.20) are somewhat suspect when applied to a slowly varying street.

A third relation between the street and the body comes from requiring that the vorticity generated and shed at the surface of the body is convected into the wake. In principle, an unsteady inviscid model might provide some quantitative information regarding the shedding of vorticity (which, incidentally, could then be used for estimates of the fluctuating lift). This possibility is not pursued here; instead, it is chosen to put

$$
\frac{\Gamma}{l}\left(U_{0}-U_{\mathrm{s}}\right)=\frac{1}{2} \delta U_{0}^{2},
$$

where $\delta$ is essentially the fraction of vorticity in the shear layers that ends up in the fully developed vortices times the square of the ratio of the velocity at the edge of the shear layer to the velocity of the free stream (see Roshko 1954). Assuming that all shed vorticity ends up in the vortices, the Kirchhoff free-streamline theory predicts $\delta=1$, but, as pointed out by Roshko (1954), in the real flow vorticity is shed at a considerably faster rate and values of $\delta$ in the vicinity of 1.75 would be more realistic. However, owing to annihilation, not all of the shed vorticity ends up in the vortices; indirect experimental observations indicate that only about $60 \%$ (Fage \& Johansen 1927), 43\% (Roshko 1954), $67 \%$ (Bloor \& Gerrard 1966), or $25 \%$ (Davies 1976) of the vorticity survives, and theoretical estimates that have been given are $50 \%$ by Prandtl (see Birkhoff 1953), 85\% (Clements 1973), and 85\% (Sarpkaya 1975) (the last two results are based on inviscid models and are probably too large). Multiplying 1.75 by a fraction of this order gives a value for $\delta$ in the vicinity of unity (probably slightly less).

Finally, the three relations (2.11), (2.20), and (2.21) may be non-dimensionalized, giving

$$
\begin{aligned}
\frac{\kappa \hat{U}_{0}^{2}}{2 \beta} C_{\mathbf{D}} & =\kappa\left(\hat{U}_{\mathbf{0}}-\hat{U}_{\mathbf{s}}\right)+\hat{D}^{\prime}, \\
(1-\epsilon) \frac{\kappa \hat{U}_{0}^{2}}{2 \beta} C_{\mathbf{D}} & =\hat{T}-\kappa \hat{U}_{\mathrm{s}}, \\
\hat{U}_{0}-\hat{U}_{\mathrm{s}} & =\frac{1}{2} \delta \hat{U}_{0}^{2},
\end{aligned}
$$

where

$$
\left.\begin{array}{c}
\kappa=\frac{h}{l}, \quad \hat{U}_{0}=\frac{l}{\Gamma} U_{0}, \quad \hat{U}_{\mathrm{s}}=\frac{l}{\Gamma} U_{\mathrm{s}}, \quad \hat{D}^{\prime}=\frac{l}{\Gamma^{2}} D^{\prime} \\
\hat{T}=\frac{l}{\Gamma^{2}} T, \quad C_{\mathrm{D}}=\frac{D}{\frac{1}{2} U_{0}^{2} b}, \quad \beta=\frac{h}{b} .
\end{array}\right\}
$$

Here $b$ is the 'breadth' of the body which is used in the definition of drag coefficient $C_{\mathrm{D}}$. Note that $\hat{U}_{\mathrm{s}}, \hat{D}^{\prime}$, and $\hat{T}$ are functions of $\kappa$ and $\alpha \equiv A / l^{2}$ alone (where $A$ is the dimensional vortex area), and are properties of the street, which for the uniform vorticity model were given numerically in Saffman \& Schatzman (1981) and Schatzman (1981). This data has been curve-fit; the results are presented in the appendix. Using $(2.22 c)$ to eliminate $\hat{U}_{0}$, and plugging in the known functions $\hat{U}_{\mathrm{s}}(\kappa, \alpha), \hat{D}^{\prime}(\kappa, \alpha)$, and $\hat{T}(\kappa, \alpha)$, two simultaneous equations for $\kappa$ and $\alpha$ result, with $C_{\mathrm{D}} / \beta, \epsilon$ and $\delta$ as parameters. Note that since $(2.22 c)$ is quadratic in $\theta_{0}$ there are in general two solutions. 


\section{Numerical results}

Given values for $C_{\mathrm{D}} / \beta, \epsilon$ and $\delta$, which are expected to be functions of the shape of the body and the Reynolds number, all the remaining quantities (which completely describe the street properties) can be determined. Quantities of particular interest are $\kappa, \alpha$, the Strouhal number

$$
S=\frac{U_{0}-U_{\mathbf{s}}}{l} \frac{b}{U_{0}}=\frac{{\hat{\theta_{0}}}_{0}-\hat{U}_{\mathrm{s}}}{\hat{U}_{0}} \frac{\kappa}{\beta}
$$

and the dimensionless vortex strength

$$
\hat{\Gamma}=\frac{\Gamma}{U_{0} b}=\frac{\beta}{\kappa \hat{U}_{0}} .
$$

The quantities $\kappa, \alpha, S \beta, \Gamma / \beta$ have been plotted in figures $2-5$ as functions of $C_{\mathrm{D}} / \beta$, for each of the two branches of solutions of $(2.22 a-c)$, for $\epsilon=0$, and for values of $\delta$ ranging from 0.6 to 1.2 (figures $2(a)-5(a)$ correspond to the larger solution for $\hat{U}_{0}$ and figures $2(b)-5(b)$ correspond to the smaller value). It should be noted that the solutions shown in figures $2(a)-5(a)$ have very small area votices, and hence the approximation of circular vortices is a good one (see Schatzman 1981). In this case, $\hat{U}_{\mathrm{s}}$ and $\hat{D}^{\prime}$ are independent of $\alpha$, and the only effect of increasing $\epsilon$ is to increase $\alpha$, all other parameters discussed above (except the energy) remain virtually unchanged. The solutions shown in figures $2(b)-5(b)$ have much larger area vortices. However, as will be pointed out, the parameters other than vortex area for these solutions do not agree with experiment. For $\delta>1$, there is no solution for $C_{\mathrm{D}} / \beta$ smaller than a certain positive critical value (the solution branches as plotted terminate, but if the two branches were plotted together, the limit-point behaviour would be obvious). To obtain reasonable values of $C_{\mathrm{D}} / \beta, \delta$ must certainly be less than 1.5 , and values closer to 1.0 are more reasonable. On the other hand, for $0<\delta<1$, there are solutions for all $C_{\mathrm{D}} / \beta$ greater than a certain negative value.

According to calculations, the independence (of quantities other than $\alpha$ ) on variations in $\epsilon$ holds even for quite large areas $\alpha$. The main effect of large values of $\epsilon$ is to terminate the solution branches when the area becomes larger than the maximum for which a steady configuration exists (for the uniform vorticity model, depending on $\kappa$, the maximum $\alpha$ ranges from about 0.25 to 0.35 ; see Saffman \& Schatzman 1981). This places a lower limit on $\kappa$ and $S \beta$ and an upper limit on $\Gamma / \beta$; for $\epsilon=0.8$, roughly $\kappa>0 \cdot 1, S \beta>0 \cdot 1, \mathrm{f} / \beta<3.5$, and for $\epsilon=0.9$, roughly $\kappa>0 \cdot 2$, $S \beta>0 \cdot 17, \hat{\Gamma} / \beta<2 \cdot 5$ for $\delta$ between 0.6 and $1 \cdot 2$. Near this cut-off point, the change from figures $2(a)-5(a)$ is very small. For the second branch of solutions (figures $2 b-5 b$ ), increasing $\epsilon$ has a similar effect, except that since the solutions $\alpha$ for $\epsilon=0$ are already quite large, there is only a gradual effect on $\alpha$. There does not appear to be available any experimental data that would provide an estimate of $\epsilon$, but to show the effect on $\alpha$ in figure $3(a)$ the corresponding quantities with $\epsilon=0.8$ have been plotted in figure 6.

For comparison, some numerical values for $\beta, \delta, \kappa, \alpha, S \beta$, and $\Gamma / \beta$ that have been obtained experimentally by other researchers are listed in table 1 and have been indicated in figures 2, 4, and 5. As has been pointed out by Hooker (1936), Timme (1957), Schaefer \& Eskinaze (1959), Berger (1964), Gerrard (1965), Davies (1976) and other authors, the typical hot-wire measurements (which give the best data currently available) are subject to certain systematic errors. These workers have tried to 

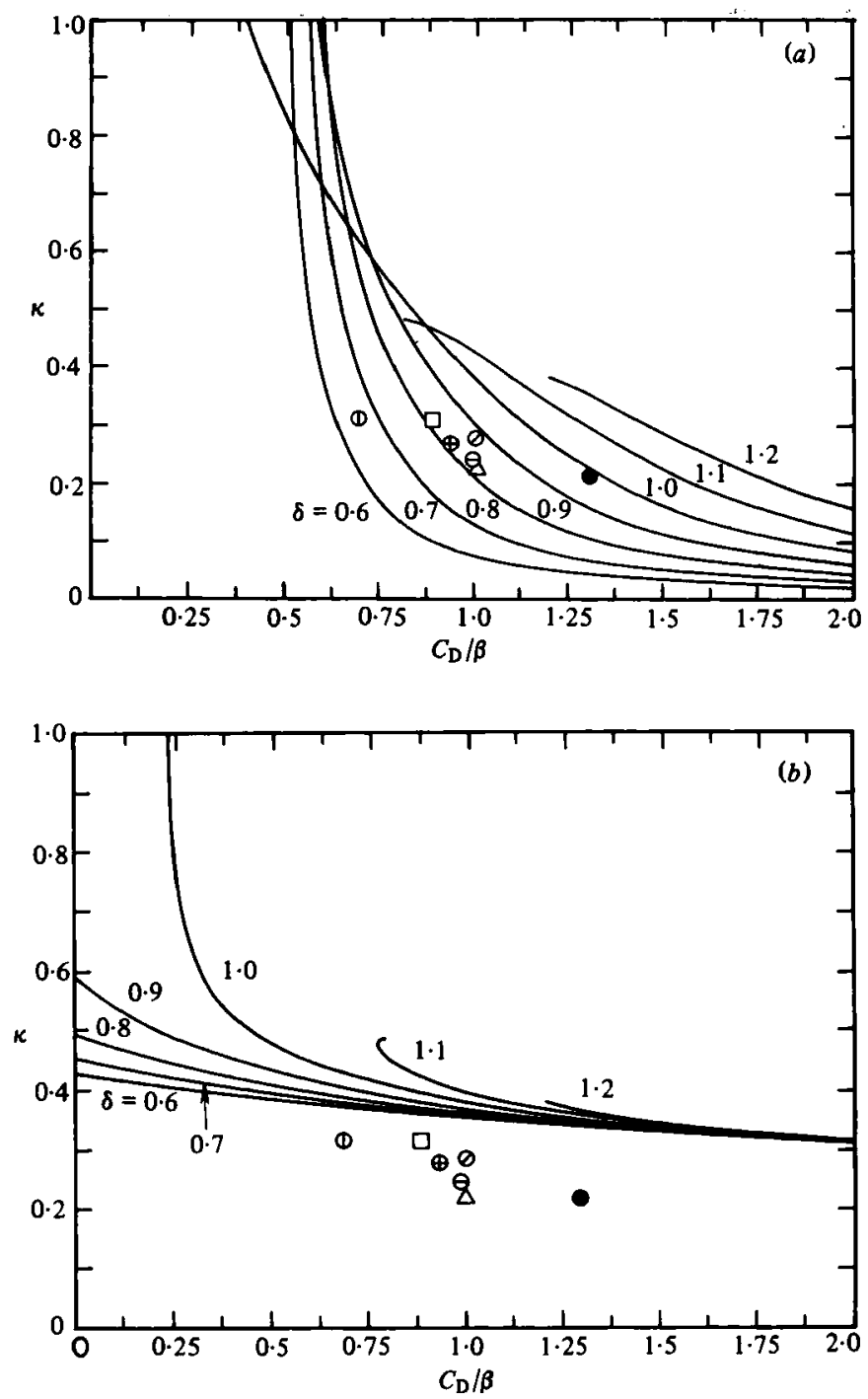

Fioure 2. Spacing ratio of the street as a function of drag coefficient for various shedding fractions $\delta$. Solutions represented by $(a)$ have the larger root $\hat{\theta}_{0}$ and those by $(b)$ have the smaller. Symbols indicate experimental data listed in table 1 .

improve the results, most recently by attempting to fit the observed data to specific vortex-street models (e.g. a street of Hamel-Oseen vortices) and in this indirect way determine the quantities of interest. It is our opinion that hot-wire measurement techniques, with the attendant problems of nonlinearity, noise and poor frequency response, are still inadequate to determine accurately the vorticity distribution within the street, and hence reported values of vortex strength, size, and transverse spacing all are subject to serious doubt. As pointed out by Gerrard (1965), the phenomena are also very sensitive to turbulence of the free stream, but in our opinion, better experimental techniques need to be developed before the discrepancies between the various experimental results can be satisfactorily explained and this matter conclusively settled. 

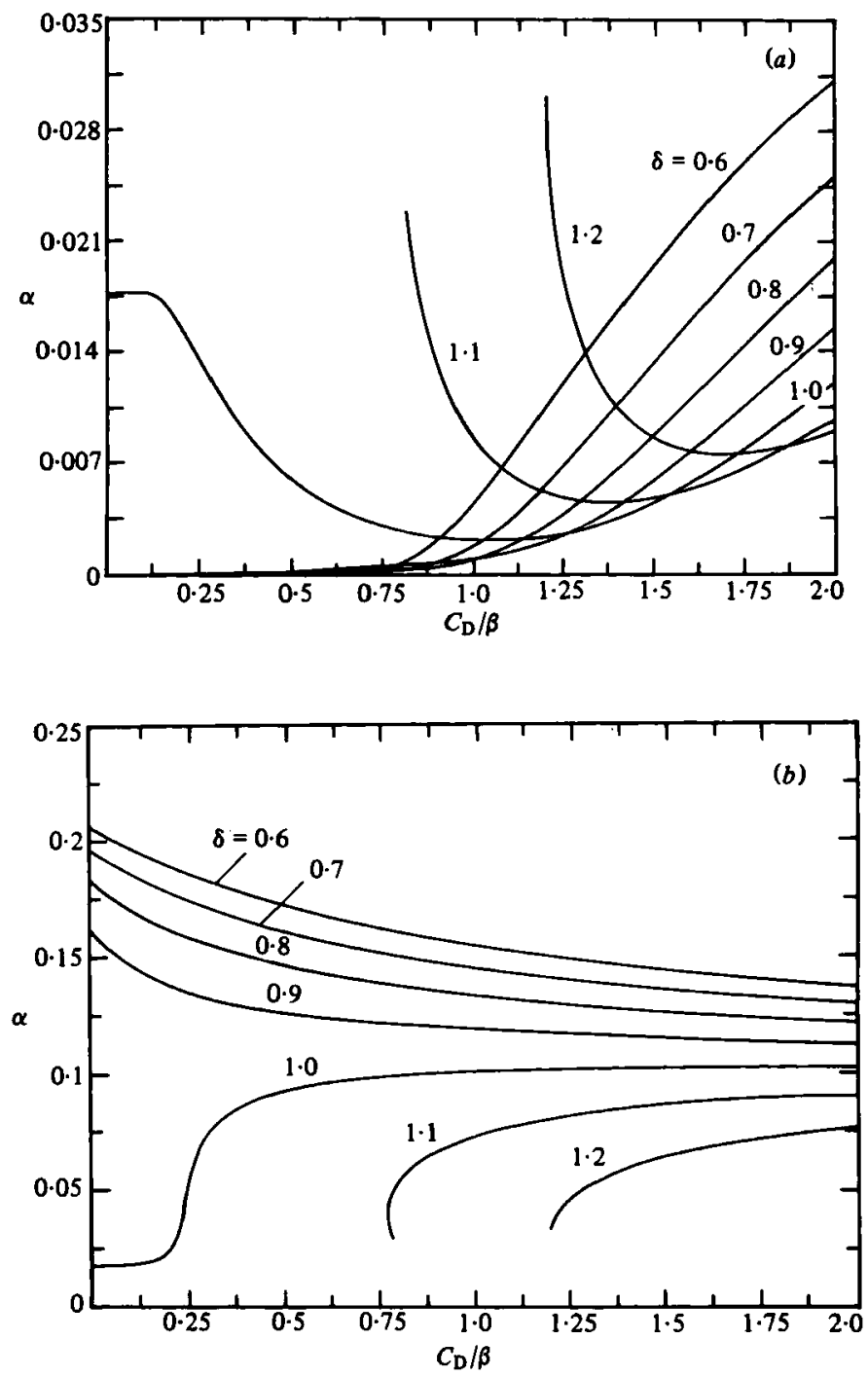

Figure 3. Vortex area as a function of drag coefficient for various shedding fractions $\delta$. Solutions represented by $(a)$ have the larger root $\hat{\theta}_{0}$ and those by $(b)$ have the smaller.

It should be noted that mean velocity profiles

$$
\bar{u}(y)=\frac{1}{l} \int_{0}^{l} u(x, y) d x,
$$

can be obtained from the published figures of shapes in Saffman \& Schatzman (1981) and Schatzman (1981) by using the relation

$$
\frac{d \bar{u}}{d y}=-\frac{1}{l} \int_{0}^{l} \omega d x=-\frac{\Gamma}{A} \bar{l}(y),
$$

where $\bar{l}(y)$ is the fraction of line $y=$ const that intersects the vortices of positive sense minus the corresponding fraction for vortices of negative sense. Unfortunately, the available experimental data on mean profiles are not adequate to make this 

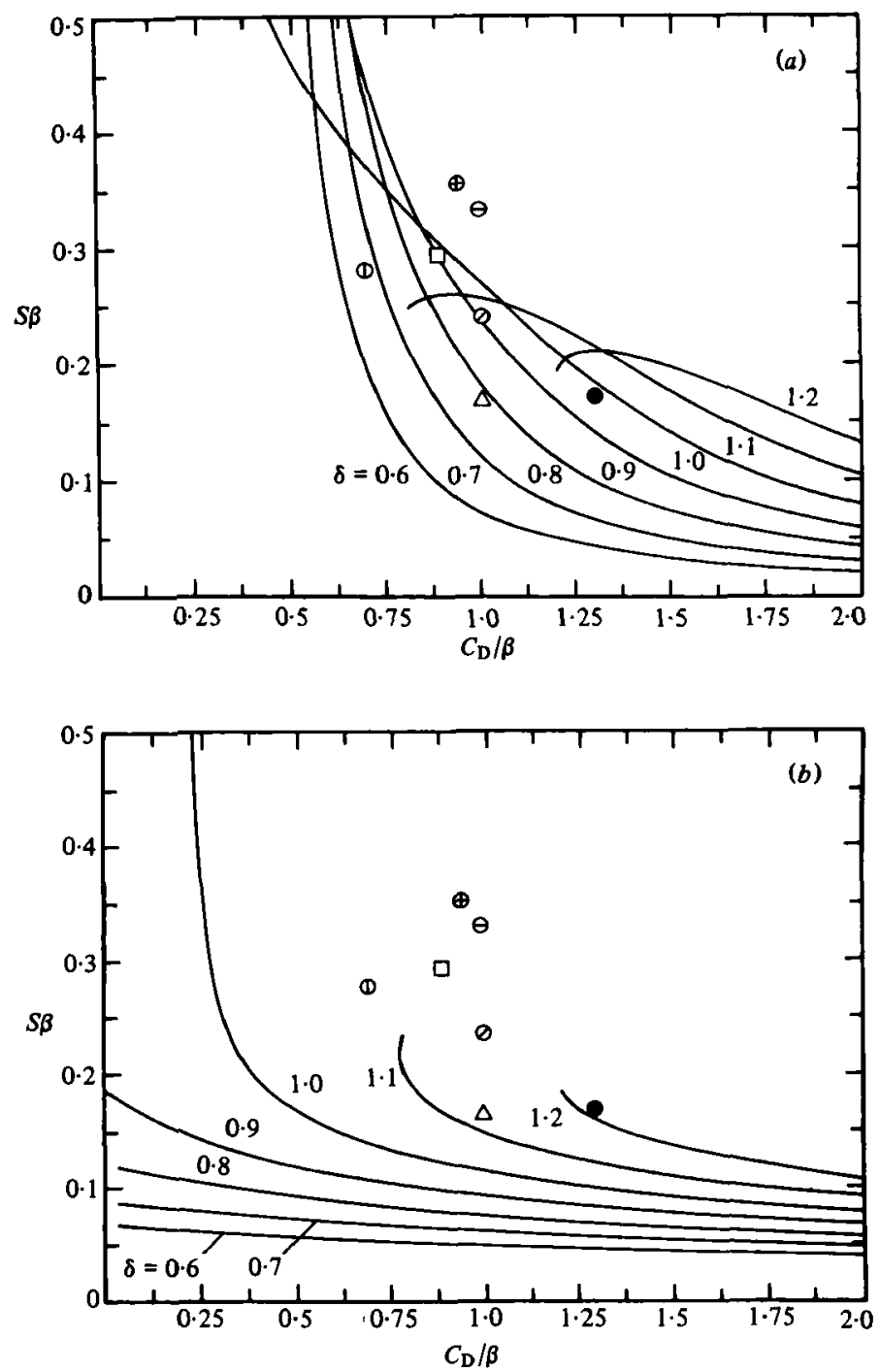

Figure 4. Wake frequency as a function of drag coefficient for various shedding fractions $\delta$. Solutions represented by $(a)$ have the larger root $\hat{U}_{0}$ and those by $(b)$ have the smaller. Symbols indicate the experimental data listed in table 1.

comparison useful. Also, it is likely that comparisons based on the uniform vorticity model will not be as satisfactory as those for models based on more realistic vorticity distributions as in Hooker (1936), Timme (1957), Schaefer \& Eskinaze (1959), Berger (1964) and Davies (1976).

Assuming that the published experimental work is not far wrong, then it is apparent from figures $2(b)-5(b)$ that this class of solutions of the conservation equations gives values of spacing ratio $\kappa$ and vortex strength $\Gamma$ that are too large, and values of Strouhal number $S$ that are too small. The configurations corresponding to figures $2(a)-5(a)$, however, are in rough agreement for these three quantities. This tends to indicate that it is the first class of solutions that correspond to the real phenomena. However, the corresponding vortex areas are smaller than the experi- 

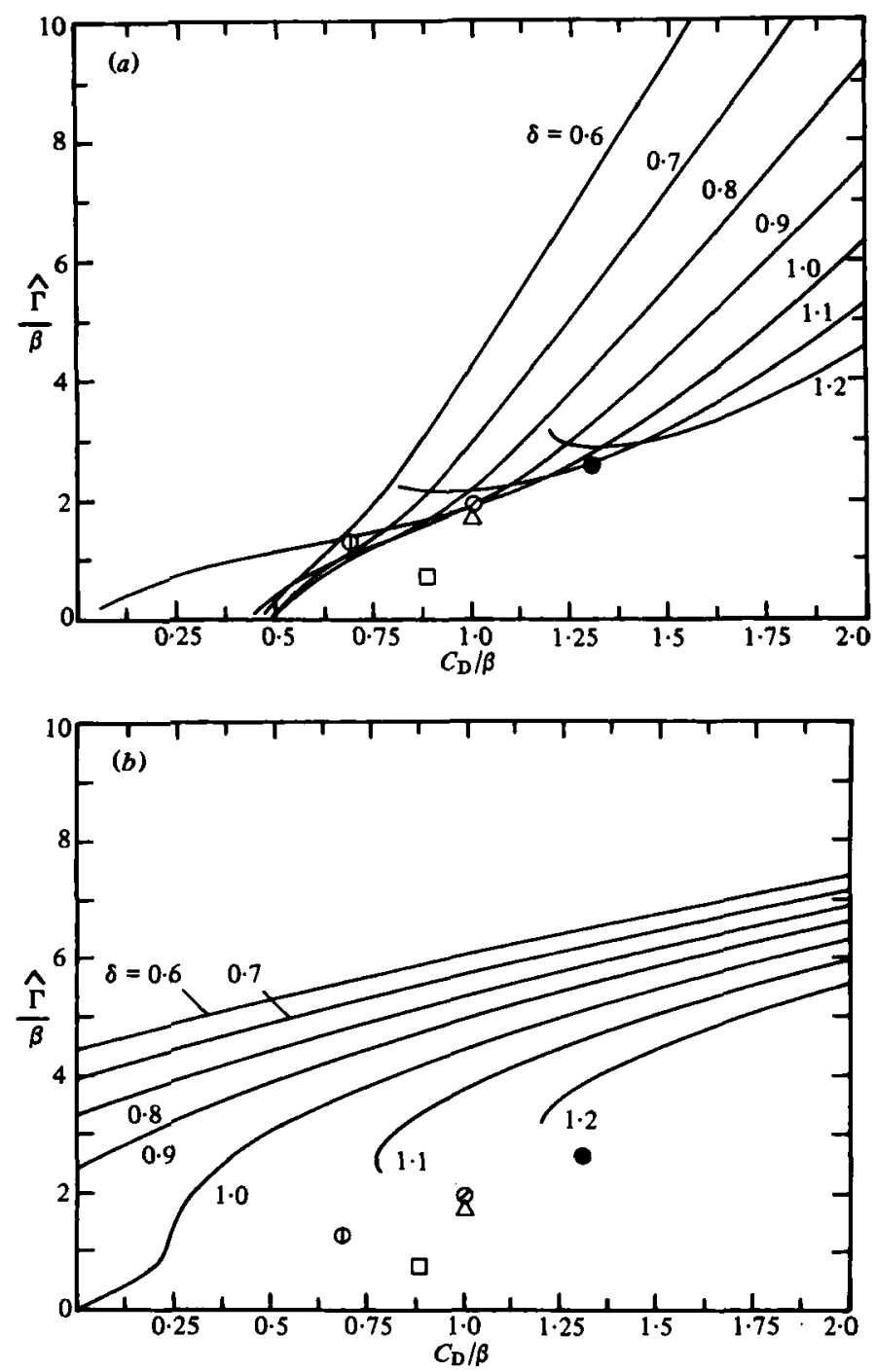

Fiqure 5. Vortex strength as a function of drag coefficient for various shedding fractions $\delta$. Solutions represented by $(a)$ have the larger root $\hat{V}_{0}$ and those by $(b)$ have the smaller. Symbols indicate the experimental data listed in table 1.

ments indicate. This suggests that there is significant energy dissipation in the real flow; this seems reasonable since laminar streets are observed only for Reynolds numbers less than roughly 150 (based on the full breadth of the body), where viscous effects still have importance in fairly large regions of the flow, and for larger Reynolds numbers the resulting turbulence probably has a significant effect in extracting energy from the flow (and hence permitting expansion and weakening of the vortices). However, the quantity $\epsilon$, which is really the only parameter in this model that is intended to simulate directly some of the effects of viscosity, does not have much effect except on the calculated vortex area. Therefore, it seems reasonable that figures $2(a), 4(a)$ and $5(a)$ represent an adequate approximation of the real flow, but the present determination of vortex area is quite indefinite. The areas may also be sensitive to the assumption of uniform vorticity. 


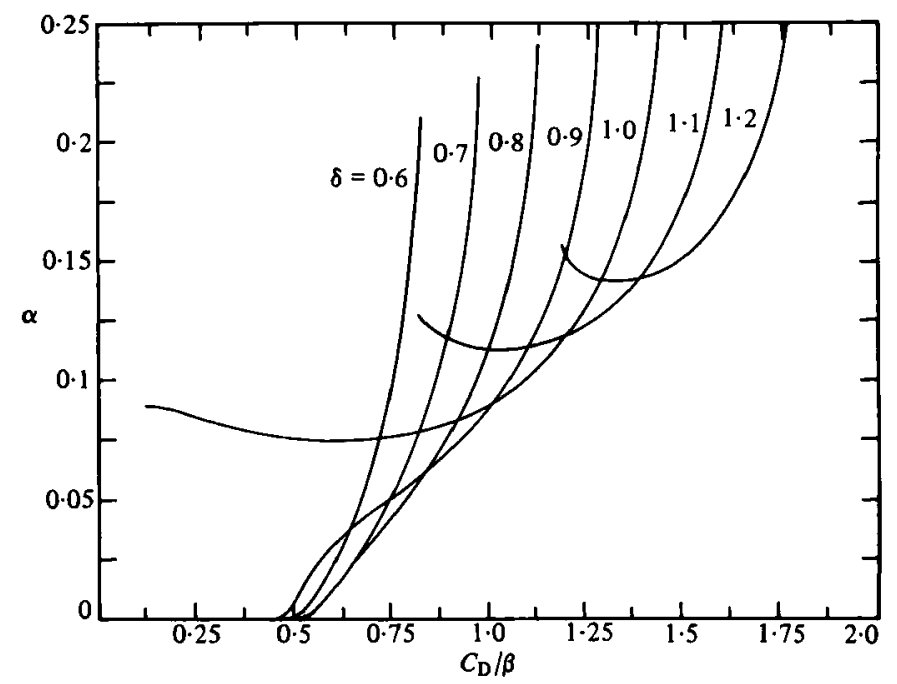

Figure 6. Vortex area as a function of drag coefficient for various shedding fractions $\delta$. Similar to figure $3(a)$, except that $\epsilon=0.8$ instead of $\epsilon=0$. Note that there are no solutions for $C_{\mathrm{D}}$ greater than certain critical values.

\begin{tabular}{|c|c|c|c|c|c|c|c|c|c|c|}
\hline Source & $R$ & $\begin{array}{l}\text { Cylinder } \\
\text { shape } †\end{array}$ & $\beta$ & $\delta$ & $\kappa$ & $\alpha$ & $\boldsymbol{S} \boldsymbol{\beta}$ & $\mathrm{\Gamma} / \beta$ & \multicolumn{2}{|c|}{$C_{\mathrm{D}} / \beta \ddagger$ Symbol } \\
\hline Hooker (1936) & 324 & $\mathbf{e}$ & 1.35 & 0.40 & 0.31 & 0.11 & $0 \cdot 29$ & $0 \cdot 70$ & $0 \cdot 89$ & $\square$ \\
\hline Timme (1957) & 200 & c & 0.95 & 0.9 & 0.21 & $0 \cdot 12$ & $0 \cdot 17$ & $2 \cdot 6$ & $1 \cdot 3$ & 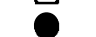 \\
\hline Schaefer \& & 62 & $\mathrm{c}$ & 2.00 & $0 \cdot 685$ & 0.31 & 0.507 & 0.28 & 1.23 & 0.69 & (1) \\
\hline \multirow[t]{2}{*}{ Eskinaze (1959) } & 94 & c & $1 \cdot 30$ & - & 0.24 & 0.212 & $0 \cdot 33$ & - & 0.99 & $\theta$ \\
\hline & 118 & c & $1 \cdot 34$ & - & $0 \cdot 27$ & $0 \cdot 114$ & 0.35 & - & 0.94 & $\oplus$ \\
\hline Berger (1964) & 151 & c & $1 \cdot 39$ & 0.785 & 0.28 & $0 \cdot 152$ & 0.238 & 1.65 & 1.0 & $\varnothing$ \\
\hline Davies (1976) & $\begin{array}{l}7 \times 10^{3}- \\
4 \times 10^{-5}\end{array}$ & d & $1 \cdot 26$ & $0 \cdot 624$ & $0 \cdot 21$ & 0.084 & $0 \cdot 165$ & 1.9 & $1 \cdot 0$ & $\triangle$ \\
\hline
\end{tabular}

$\dagger \mathrm{c}=$ Circular cylinder, $d=$ Semicircular cylinder, flat surface normal to free stream and facing upstream, e = Elliptical cylinder, major axis aligned with free stream.

$\ddagger$ The data for $C_{\mathrm{D}}$ were not provided by the researchers cited, but is appropriate data obtained from Tritton (1959) and Roshko (1961).

TABLE 1. Calculated vortex-street parameters based on published experimental data. Data pertaining specifically to wake were taken at the estimated point downstream where the vortex street was first fully formed.

\section{Slow evolution of the street}

Despite the assumptions of the analysis of $\S 2$ in the derivation of (2.19) that the vortex street does not evolve, it is perhaps instructive to examine how the model might be used to approximate a slowly varying (pseudo-steady) wake. Along with the viscous diffusion of the vortices, in the real flow there is an accompanying energy dissipation and annihilation of vorticity. Hence, it would seem reasonable to observe the effect of increasing $\epsilon$ and decreasing $\delta$. Figures 7-9 were calculated for fixed $C_{\mathrm{D}}$ (as pointed out in $\S 2$, this corresponds to fixed momentum flux in the wake) and $\epsilon=0$ by specifying the initial value of $\beta$ (i.e. fixing the initial width of the street), solving 
(2.22), and thereafter allowing $\beta$ to change while $\delta$ is decreased and $S$ is held constant. This last condition is necessary so that vortices be conserved as the wake evolves, since the number of vortices per unit time passing a point in the wake fixed relative to the body is $\left(U_{0}-U_{\mathrm{s}}\right) / l$ and $S=\left[\left(U_{0}-U_{\mathrm{s}}\right) / l\right]\left[b / U_{0}\right]$. Note that for the physically reasonable class of solutions of $(2.22) \epsilon$ has essentially no effect save on the vortex area. Thus, under these changes, the model would predict a change in the configuration of the street as indicated in figures 7-9 for $C_{\mathrm{D}}=1,1 \cdot 35,1 \cdot 7$ due to decreasing $\delta$ (with $\epsilon=0$ ), along with an additional increase in area $\alpha$ due to increasing $\epsilon$, which is not taken into account. Presumably, the corresponding increase in $\epsilon$ to describe the real flow adequately would be sufficient to result in a net increase of $\alpha$, although a quantitative study of the relationship between $\epsilon$ and $\delta$ would be helpful in completing this analysis. This approximate evolution model is very crude, but figures 7-9 do clearly show the increase in transverse spacing $\beta$ and spacing ratio $\kappa$ that is remarked in practically every experimental report on the Kármán vortex street at low Reynolds number (see Hooker 1936). The model also predicts the nearly constant streamwise vortex spacing $l$ that is observed (see e.g. Schaefer \& Eskinaze 1959). Lacking complete and accurate experimental results, and lacking a theory to predict values of $\epsilon$ and $\delta$ during the evolution, a more serious comparison is not possible at this time.

\section{Street stability}

An important question is whether or not the stability properties of the steady vortex street model are relevant. Since von Kármán first studied the stability of the point vortex street (von Kármán 1911), much use has been made by researchers of his result that the spacing ratio $\kappa=0 \cdot 28055$. . gives the least unstable configuration (linearly neutral). It is our belief that the stability properties of the steady street either are not relevant to the formation process of the real street or at least any connection is still obscure. A more reasonable hypothesis is that the stability properties have some relevance to the slow street-evolution process, after the street has fully developed. The observed rearrangement process for the laminar case, below Reynolds number about 160 (see Taneda 1959; Matsui \& Okude 1980), bears a strong resemblance in its initial stages to the normal modes from the linear stability analysis. The hypothesis is that the evolution process is slow enough that the properties of periodic wakes are of good approximation, and that a street which at first lies within the band of stability (see Saffman \& Schatzman (1982) for a discussion of the stability for the uniform vorticity model) becomes unstable as the vortex area $\alpha$ and spacing ratio $\kappa$ change. Quantitative verification of this hypothesis using data such as presented in figures 7-9 is not feasible, since the calculated vortex areas for these configurations are too small (as commented upon above). However, the uniform vorticity model does predict that a stable configuration will become unstable as $\kappa$ is increased much above $\mathbf{0 . 3 5}$, independent of the vortex area.

The stability calculation describes disturbances proportional to $e^{\sigma t}$, where $\sigma$ is non-dimensionalized by $l^{2} / \Gamma$, which is not a convenient scale. A more useful measure of growth rate is the growth in one period of the oscillatory motion of the wake (i.e. time $\left.l /\left(U_{0}-U_{\mathrm{s}}\right)\right)$. Then

$$
\sigma t=\frac{\Gamma}{l^{2}} \hat{\sigma} t=\frac{\Gamma}{l^{2}} \hat{\sigma} \frac{l}{U_{0}-U_{\mathrm{s}}} \hat{t}=\frac{\Gamma / \beta}{S \beta} \kappa^{2} \hat{\sigma} \hat{t} \equiv \sigma^{*} \hat{t}
$$

The exponential growth rates $\sigma^{*}$ have been plotted versus $\kappa$ for several different vortex areas in figure 10 . It has been assumed that $\Gamma / \beta=1.5$ and $S \beta=0.25$ 


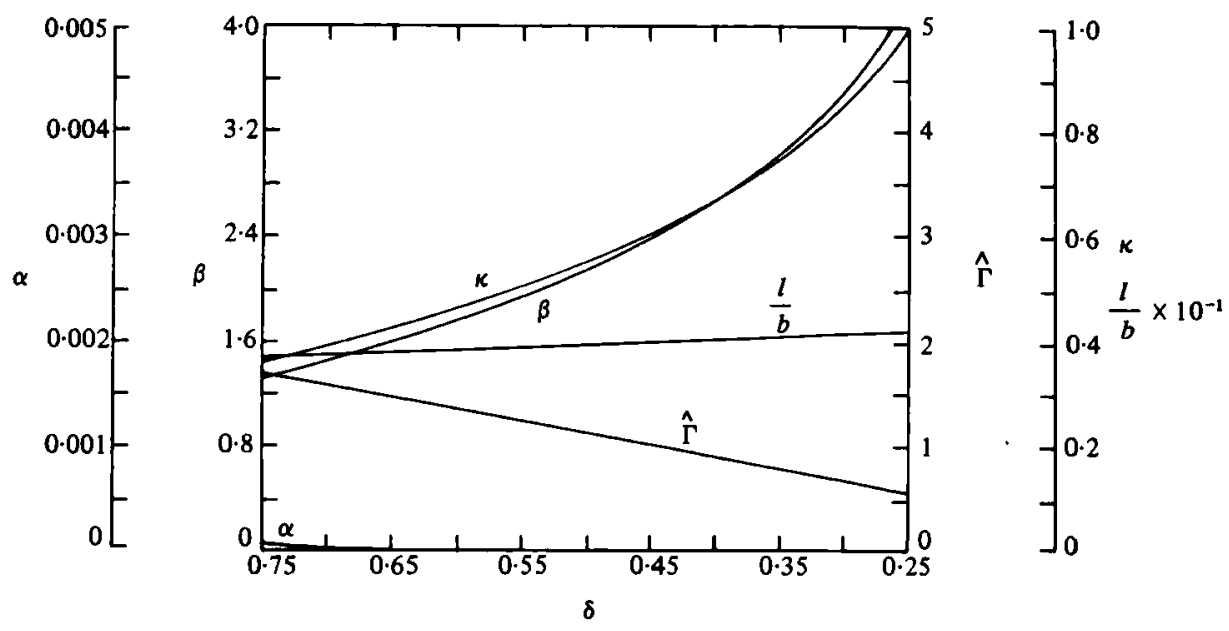

Fiaure 7. Model results for the slow evolution of the street as vorticity is lost ( $\delta$ decreases) for $C_{\mathrm{D}}=1, S=0 \cdot 222$. This configuration corresponds to Reynolds number about 1000 (see Roshko 1961).
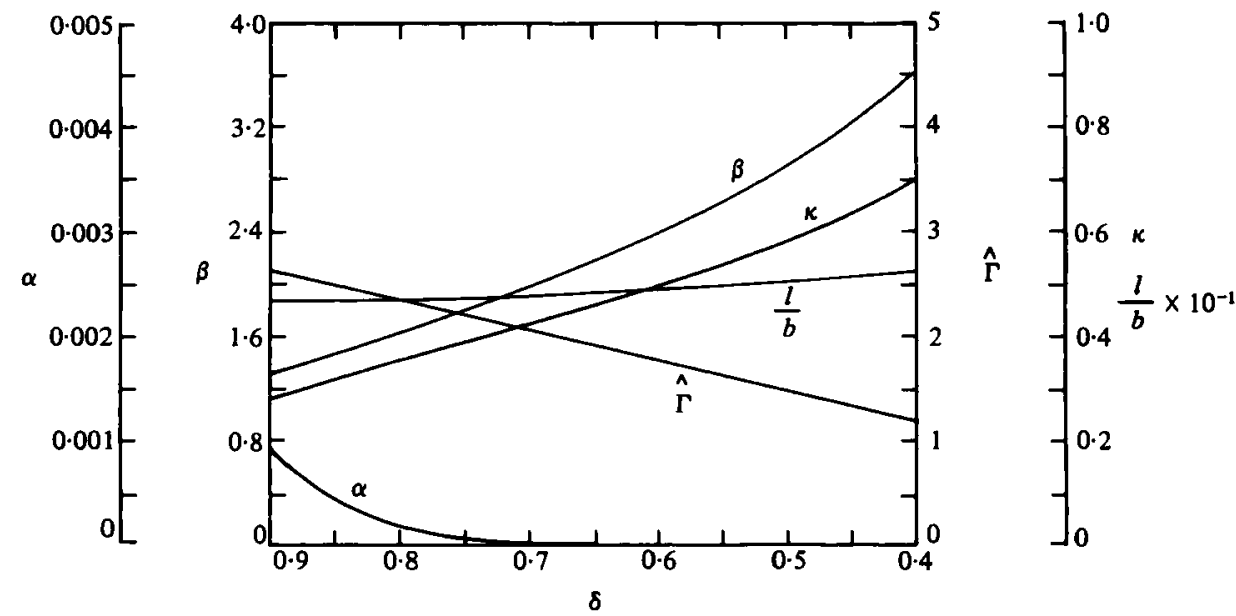

Figure 8. Model results for the slow evolution of the street as vorticity is lost ( $\delta$ decreases) for $C_{\mathrm{D}}=1.35, S=0.172$. This configuration corresponds to Reynolds number about 120 (see Roshko $1961)$.

independent of $\kappa$ (these are typical experimental values). For comparison, note that a value $\sigma^{*}=1$ implies that a small disturbance will grow by a factor of 10 in $2 \cdot 3$ wavelengths of the motion.

These theoretical considerations are compatible with the experimental observations of Taneda (1959) and Matsui \& Okude (1980). In particular, the latter researchers found rearrangement of the street at Reynolds number 140 beginning about 12 wavelengths downstream of the body, where $\kappa$ was observed to have grown to about $0 \cdot 5$, and the rearrangement was complete around 16 cycles later. Of course, the linear stability analysis cannot predict the rearrangement beyond its initial stages, but these 


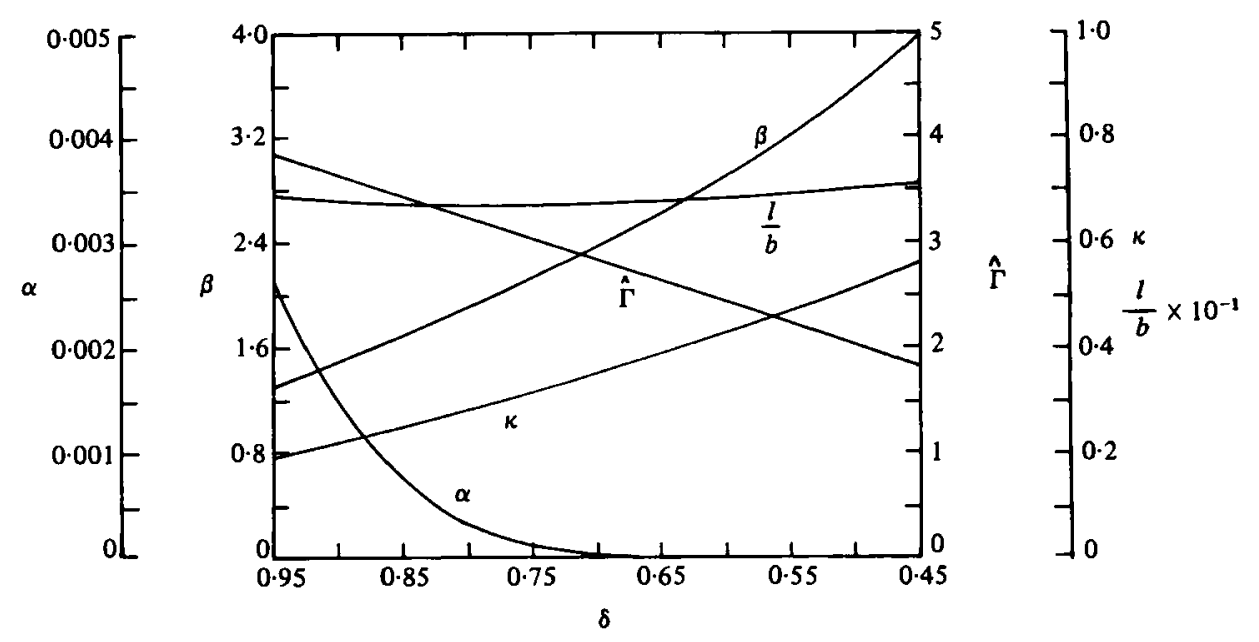

Figure 9. Model results for the slow evolution of the street as vorticity is lost ( $\delta$ decreases) for $C_{\mathrm{D}}=1 \cdot 7, S=0 \cdot 124$. This configuration corresponds to Reynolds number about 50 (Roshko 1961 ).

numbers do make it seem reasonable that the linear stability growth rates are large enough to result in rearrangement at the point where it is observed.

It may well be that this pseudo-steady approximation, in which properties of the evolving street are approximated at each stage of evolution by those of corresponding periodic streets, is not good. However, the qualitative behaviour of this model does tend to support the hypothesis that the observed features of the rearrangement of the vortex street may in part be explained by hydrodynamic stability.

\section{Rearrangement}

An important and as-yet inconclusively answered question is whether pairing is an adequate description of the rearrangement process which the vortex street is observed to undergo. If the rearrangement process is pairing, then the wake frequency (i.e. Strouhal number) must be exactly halved. The experimental work of Taneda (1959) and Matsui \& Okude (1980) includes measurements of the ratio of streamwise spacing of the vortices before and after the rearrangement. Taneda does not report vortex frequencies, but the others report frequencies of the secondary vortex street which are not half that of the primary vortex street. The reported ratio of frequencies ranges from about 0.5 at Reynolds number $R \approx 150$ to about 0.23 at $R \approx 110$. If correct, this would indicate that the rearrangement process is not pairing, but a more general redistribution of vorticity. In the following discussion the process is assumed to be pairing, and it should be kept in mind that this may only be accurate at the higher ranges of Reynolds number (around 140-160). However, because of the large quantitative discrepancy in the ratio of streamwise spacings between the results of these two sets of experiments, we believe that further experiments need to be conducted before this matter can be settled.

The inviscid model may be used to predict the ratio of streamwise spacings as follows. As pointed out previously, the fact that the momentum flux in the wake must be constant implies that (2.11) is approximately invariant in the nearly uniform parts 


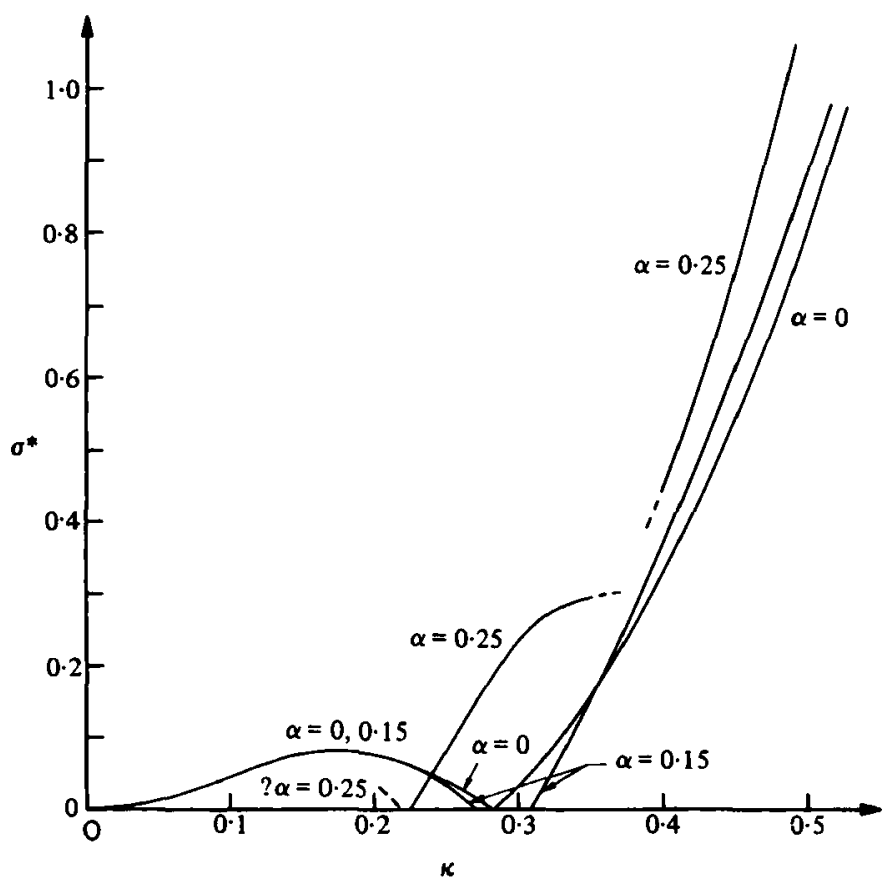

Figure 10. Growth rate of small perturbations to the finite-area uniform-vorticity street (normalized by the wake frequency). $\hat{\Gamma} / \beta=1.5$ and $S \beta=0.25$ have been assumed. A value $\sigma^{*}=1$ implies that there are disturbances that grow by a factor of 10 in $2 \cdot 3$ cycles of the wake. The behaviour of $\sigma^{*}$ for $\alpha=0.25$ is uncertain below $\kappa=0.225$ and near $\kappa=0.375$.

of the wake. Suppose that after rearrangement the vortices have streamwise spacing $l^{\prime}=\lambda l$ and strength $\Gamma^{\prime}=\mu \Gamma$. The condition that $(2.11)$ is invariant when the new Strouhal number is $S^{\prime}=0.5 S$ gives $\lambda$ as a function of $\mu, S, \kappa, \beta, \alpha$, and $\alpha^{\prime}$. To reasonable accuracy the circular-vortex approximation may be used (eliminating the dependence on $\alpha$ and $\alpha^{\prime}$ ). Invariance of (2.11) while $U_{0}$ is held constant then implies

$$
\frac{1}{\pi}+\kappa \tanh \pi \kappa\left[\frac{1}{1-\beta S / \kappa}-2\right]=\frac{\mu^{2}}{\lambda}\left\{\frac{1}{\pi}+\kappa^{\prime} \tanh \pi \kappa^{\prime}\left[\frac{1}{1-\frac{1}{2} \lambda \beta S / \kappa}-2\right]\right\}
$$

where $\kappa^{\prime}$ (the spacing ratio after rearrangement) is determined by the relation

$$
\frac{\tanh \pi \kappa}{1-\beta S / \kappa}=\frac{\mu}{\lambda} \frac{\tanh \pi \kappa^{\prime}}{1-\frac{1}{2} \lambda \beta S / \kappa} .
$$

Equations (6.1) and (6.2) may be solved for $\kappa^{\prime}$ and $\lambda$ as functions of $\mu, S, \kappa$ and $\beta$. A typical set of solutions have been plotted as functions of $\kappa$ for several values of $S$ and $\beta=2 \cdot 5, \mu=2,1 \cdot 5,1$ (no circulation loss, $25 \%$ loss, and $50 \%$ loss, respectively) in figures $11(a, b)$. It should be noted that in all cases the final spacing ratio $\kappa^{\prime}$ is smaller than the initial, and this decrease can be very substantial. This indicates that vortex streets with $\kappa>0 \cdot 4$, which should be very unstable according to $\$ 5$, can rearrange themselves to form stable or more nearly stable configurations. The solution curves depend strongly on $\beta$ (which is unknown except to rough approxi- 

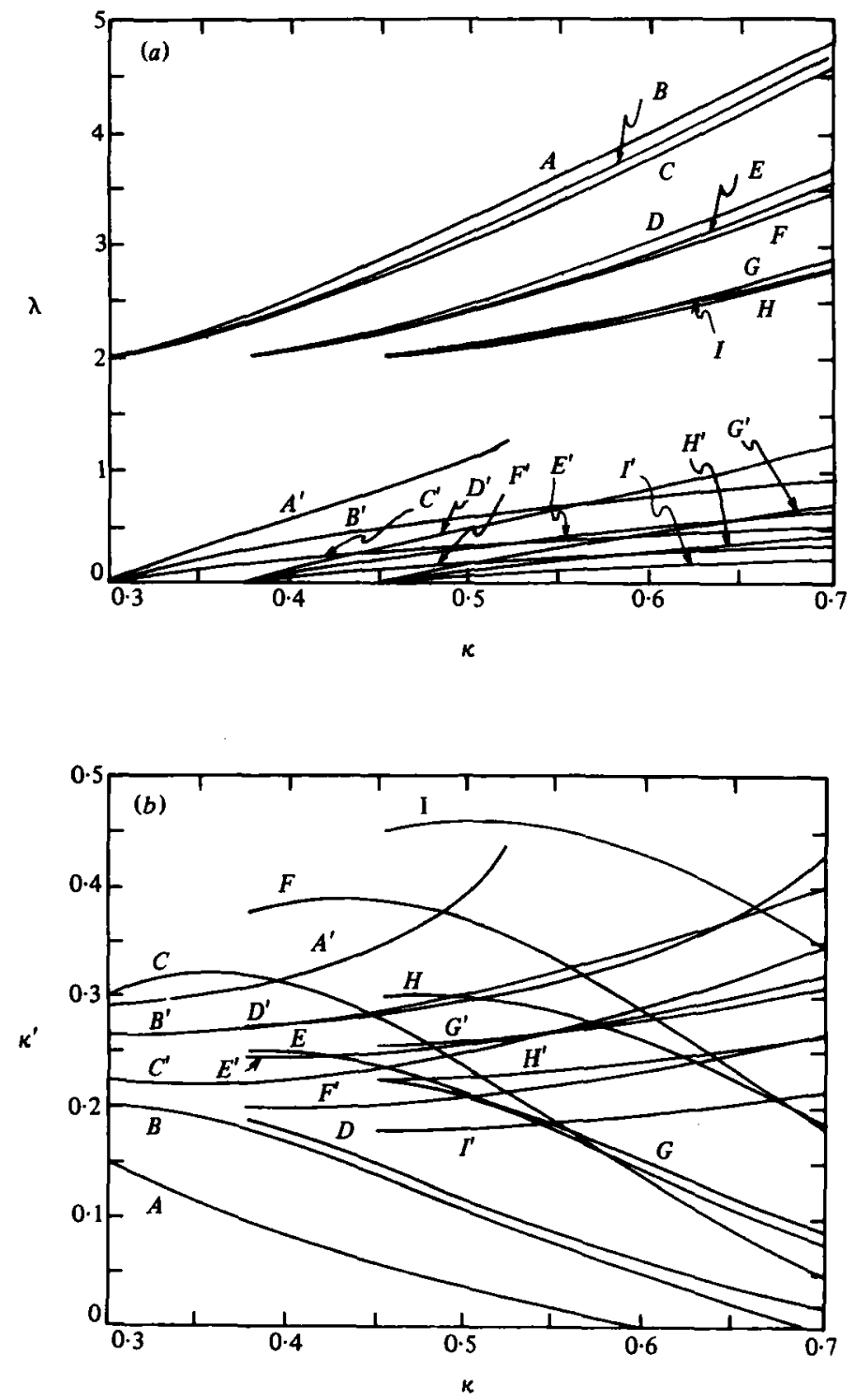

Figure 11. Ratio $\lambda$ of streamwise vortex spacings after to those before rearrangement and final spacing ratio $\kappa^{\prime}$ versus initial spacing ratio $\kappa$ for $\beta=2 \cdot 5$. Curves $A-C$ have $S=0 \cdot 12, D-F$ have $S=0 \cdot 15, G-I$ have $S=0 \cdot 18, A, D, G$ have no vorticity loss; $B, E, H$ have $25 \%$ loss; $C, F, I$ have $50 \%$ loss. Primed and unprimed letters denote the two solutions of the equations for a given set of parameters.

mation), but a comparison with experiment was attempted as follows. The 'Roshko line' giving $S$ as a function of Reynolds number for the circular cylinder (with which Matsui \& Okude claim agreement) was assumed (see Roshko 1953). Figure 12 shows the larger solution resulting from this model for $\kappa=0.4,0.5,0.6, \beta=2.4, \mu=2$, together with the experimental data (curve fit) of the aforementioned researchers (for which $\kappa, \beta$, and $\mu$ are unknown). Decreasing $\mu$ to 1 has a small effect on $\lambda$ in all cases (generally $\lambda$ decreases slightly). It is evident that the experimental data can be 


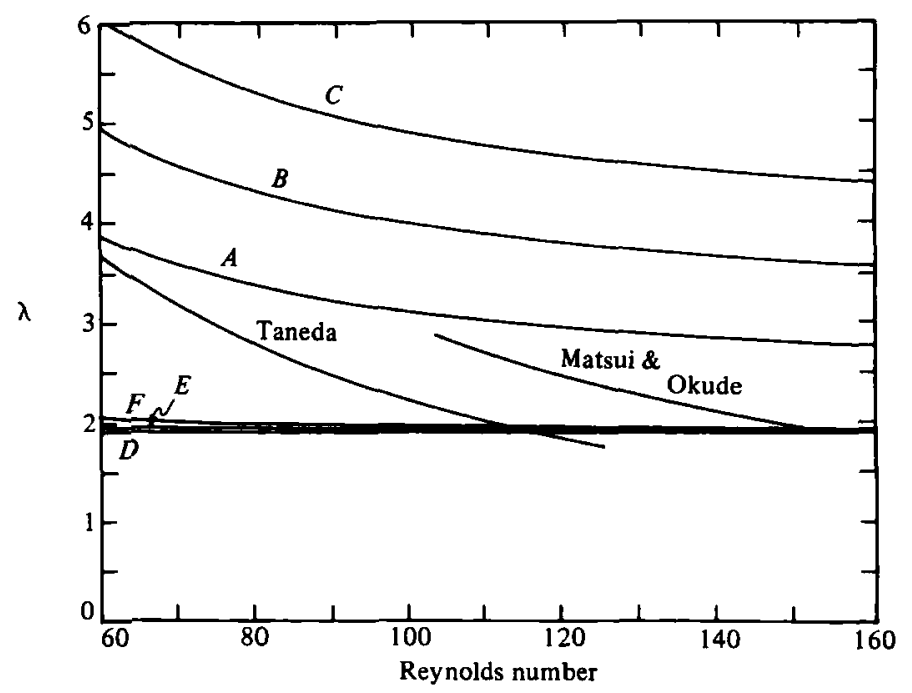

Figure 12. The solution of larger $\lambda$ of the rearrangement equations versus Reynolds number for $\mu=2$ (vorticity is conserved) and where the 'Roshko line' has been used to obtain the Strouhal number. Curves $A-C$ have $\beta=2 ; D-F$ have $\beta=4 ; A, D$ have $\kappa=0.4 ; B, E$ have $\kappa=0.5 ; C, F$ have $\kappa=0 \cdot 6$. Decreasing $\mu$ to 1 (50\% vorticity loss) has only a small effect. The experimental data of Taneda (1959) and Matsui \& Okude (1980) are indicated.

matched by picking reasonable values of $\mu, \kappa$, and $\beta$ which are functions of Reynolds number. The present model does not permit independent prediction of each of these three quantities, but only provides a semi-empirical relation between them.

This work was supported by the Department of Energy (Office of Basic Energy Sciences). We acknowledge with gratitude the granting of time by Control Data Corporation on the Cyber 203 computer at the CDC Service Center, Arden Hills, Minnesota.

\section{Appendix}

The three functions $\hat{U}_{\mathrm{s}}(\kappa, \alpha), \hat{D}^{\prime}(\kappa, \alpha)$, and $\hat{T}(\kappa, \alpha)$, representing the propagation velocity, part of the momentum, and the energy of the wake for the uniform vorticity model, were calculated numerically as deseribed in Saffman \& Schatzman (1981) and Schatzman (1981). The data was curve-fit for values of $\kappa$ between 0.1 and 0.8 . Up to the largest-area solution calculated for $\kappa$ less than about 0.36 and up to the point where the dependence of $\alpha$ develops strong curvature (greater than $90 \%$ of the maximal area) for larger $\kappa$, errors in the approximation are less than $1 \%$. For $\alpha<0 \cdot 15$, errors are typically less than $0.1 \%$. The approximate results are:

$$
\begin{aligned}
& \hat{O}_{\mathrm{s}}(\kappa, \alpha)=\frac{1}{2} \tanh \pi \kappa+a_{1} \alpha^{2}+a_{2} \alpha^{4}+a_{2} \alpha^{6}, \\
& \hat{D}^{\prime}(\kappa, \alpha)=\frac{1}{2 \pi}-\kappa \hat{U}_{\mathrm{s}}(\kappa, \alpha)+b_{1} \alpha^{2}+b_{2} \alpha^{4}+b_{3} \alpha^{6}, \\
& \hat{T}(\kappa, \alpha)=\frac{1}{2 \pi}\left[\log \frac{\cosh \pi \kappa}{(\pi \alpha)^{\frac{1}{2}}}+\frac{1}{4}\right]+c_{1} \alpha^{2}+c_{2} \alpha^{4},
\end{aligned}
$$


where

$$
\begin{aligned}
& a_{1}=\pi^{2}\left(\tanh ^{2} \pi \kappa-\frac{2}{3}\right) \sinh \pi \kappa \operatorname{sech}^{3} \pi \kappa \\
& a_{2}=-120\left(\tanh ^{2} \pi \kappa-0 \cdot 412\right)\left(1-0 \cdot 82 \frac{\cosh ^{8} \pi \kappa}{19 \cdot 54+\cosh ^{8} \pi \kappa}\right) \operatorname{sech}^{2} \pi \kappa \\
& a_{3}=95\left(\tanh ^{2} \pi \kappa-0 \cdot 35\right) \cosh ^{2} \pi \kappa \cosh ^{3} \pi \kappa \\
& b_{1}=-\frac{10}{3}\left(\tanh ^{2} \pi \kappa-\frac{2}{3}\right)^{2} \\
& b_{1}=-\frac{65}{2}\left(\tanh ^{2} \pi \kappa-0 \cdot 26\right)\left(1-0 \cdot 847 \frac{\sinh ^{8} \pi \kappa}{\frac{2}{3}+\sinh ^{8} \pi \kappa}\right) \\
& b_{3}=25\left(1-0 \cdot 7 \frac{\sinh ^{8} \pi \kappa}{\frac{2}{3}+\sinh ^{8} \pi \kappa}\right) \\
& c_{1}=\frac{5}{8}\left(\tanh ^{2} \pi \kappa-\frac{2}{3}\right)^{2}, \quad c_{2}=\frac{3}{5} \operatorname{sech} \pi \kappa
\end{aligned}
$$

\section{REFERENCES}

Berger, E. 1964 Die Bestimmung der hydrodynamischen Grössen einen Karmanshen Wirbelstrasse aus Hitzdrahtmessungen bei kleinen Reynoldschen Zahlen. Z. Flugwiss. 12, 41-59.

Birkhoff, G. 1953 Formation of vortex streets. J. Appl. Phys. 24, 98-103.

Bloor, M. S. \& Gerrard, J. H. 1966 Measurements of turbulent vortices in a cylinder wake. Proc. R. Soc. Lond. A 294, 319-342.

CLEmENTs, R. R. 1973 An inviscid model of two-dimensional vortex shedding. J. Fluid Mech. 57, 321-336.

Davies, M. E. 1976 A comparison of the wake structure of a stationary and oscillating bluff body, using a conditional averaging technique. J. Fluid Mech. 75, 209-231.

Fage, A. \& Johansen, F. C. 1927 The flow of air behind an inclined flat plate of infinite span. Aero. Res. Counc. $R \& M$, no. 1104, 81-106.

Gerrard, J. H. 1965 A disturbance-sensitive Reynolds number range of the flow past a circular cylinder. J. Fluid Mech. 22, 187-196.

Goldstein, S. (ed.) 1938 Modern Developments in Fluid Dynamics, vol. II, pp. 556-565. Clarendon.

Hooker, S. G. 1936 On the action of viscosity in increasing the spacing ratio of a vortex street. Proc. R. Soc. Lond. A 154, 67-89.

Kármán, T. v. 1911 Über den Mechanismus des Widerstands, den ein bewegter Korper in einer Flüssigkeit erfährt. Göttinger Nachrichten, Math. Phys. Kl., pp. 509-517.

KáRmán, T. v. 1912 Ủber den Mechanismus des Widerstands, den ein bewegter Korper in einer Flüssigkeit erfährt. Göttinger Nachrichten, Math. Phys. Kl., pp. 547-556.

Kármán, T. v. \& Rubach, H. L. 1912 Über den Mechanismus des Flüssigkeits- und Luftwiderstands. Phys. Z. 13, 49-59.

KaUfmann, W. 1951 Über den Mechanismus der Wirbelkerne einer Kármánschen Wirbelstraße. Ing. Arch. 19, 192-199.

Matsui, T. \& OKUDE, M. 1980 Rearrangement of Kármán vortex street at low Reynolds numbers. In Proc. XVth Int. Cong. Theor. Appl. Mech., University of Toronto, August 17-23, 1980, pp. 1-27.

Roshko, A. 1953 On the development of turbulent wakes from vortex streets. NACA Tech. Note no. 2913 , pp. 1-77.

Roshko, A. 1954 On the drag and shedding frequency of two-dimensional bluff bodies. NACA Tech. Note no. 3169, pp. 1-29.

Roshko, A. 1961 Experiments on the flow past a circular cylinder at very high Reynolds number. J. Fluid Mech. 10, 345-356.

Safrman, P. G. \& Schatzman, J. C. 1981 Properties of a vortex street of finite vortices. SIAM J. Sci. Stat. Comp. 2, 285-295. 
Saffman, P. G. \& Schatzman, J. C. 1982 Stability of the Kármán vortex street. J. Fluid Mech. $117,171-185$.

Sarpkaya, T. 1975 An inviscid model of two-dimensional vortex shedding for transient and asymptotically steady separated flow over an inclined plate. J. Fluid Mech. 68, 109-128.

SCHAEFer, J. \& Eskinaze, S. 1959 An analysis of the vortex street generated in a viscous fluid. J. Fluid Mech. 75, 241-260.

Schatzman, J. C. 1981 A model for the von Kármán vortex street. Ph.D. thesis, California Institute of Technology.

SChLAYER, K. 1928 Über die Stabilität der Kármánschen Wirbelstraße gegenüber beliegigen Störungen in drei Dimensionen. Z. angew. Math. Mech. 8, 352-372.

TANEDA, S. 1959 Downstream development of the wakes behind cylinders. J. Phys. Soc. Japan $14,843-848$.

Trмme, A. 1957 Über die Geschwindigkeitsverteilung in Wirbelen. Ing. Arch. 25, 205-225.

Tritton, D. J. 1959 Experiments on the flow past a circular cylinder at low Reynolds number. J. Fluid Mech. 6, 547-567.

WeIHS, D. 1973 On the existence of multiple Kármán vortex-street modes. J. Fluid Mech. 61, 199-205. 